\title{
BMJ open Antidopaminergic drugs and acute pancreatitis: a population-based study
}

\author{
Robert Bodén, ${ }^{1,2}$ Tomas S Bexelius, ${ }^{3}$ Fredrik Mattsson, ${ }^{3}$ Jesper Lagergren, ${ }^{3,4}$ \\ Mats Lindblad, ${ }^{3,5}$ Rickard Ljung ${ }^{3}$
}

To cite: Bodén R, Bexelius TS, Mattsson F, et al. Antidopaminergic drugs and acute pancreatitis: a population-based study. BMJ Open 2012;2:e000914. doi:10.1136/

bmjopen-2012-000914

- Prepublication history and additional tables for this paper are available online. To view these files please visit the journal online (http://dx. doi.org/10.1136/

bmjopen-2012-000914).

Access to data All authors, external and internal, had full access to all of the data (including statistical reports and tables) in the study and can take responsibility for the integrity of the data and the accuracy of the data analysis.

Received 22 February 2012 Accepted 4 April 2012

This final article is available for use under the terms of the Creative Commons Attribution Non-Commercial 2.0 Licence; see http://bmjopen.bmj.com

For numbered affiliations see end of article.

Correspondence to Dr Robert Bodén; robert.boden@neuro.uu.se

\section{ABSTRACT}

Objectives: To evaluate the suggested association between antidopaminergic drugs and acute pancreatitis.

Design: A large population-based nested case-control study.

Setting: Swedish nationwide study from 2006 to 2008.

Participants: The Patient Register was used to identify 6161 cases of acute pancreatitis. The 61637 control subjects were randomly selected from the Register of the Total Population by frequency-based density sampling, matched for age, sex and calendar year.

Exposure: Exposure data were extracted from the Prescribed Drug Register. Antidopaminergic drugs were grouped into antiemetic/anxiolytic and other antipsychotics. Current use of antidopaminergic drugs was defined as filling a prescription 1-114 days before index date, while previous use was 115 days to 3.5 years before index date.

Main outcome measures: Cases were defined as being diagnosed as having acute pancreatitis. ORs and $95 \% \mathrm{Cls}$ were calculated using unconditional logistic regression.

Results: The unadjusted OR indicated an increased risk of acute pancreatitis among current users of antiemetic/anxiolytics (OR 1.9, 95\% Cl 1.4 to 2.6 ), but not in the multivariable model adjusting for alcoholrelated comorbidity, chronic obstructive lung disease, ischaemic heart disease, obesity, diabetes, opioid use, gallstone disease, educational level, marital status and number of concomitant medications (OR $0.9,95 \% \mathrm{Cl}$ 0.6 to 1.2 . Similarly, among current users of other antipsychotics, the unadjusted OR was $1.4(95 \% \mathrm{Cl} 1.1$ to 1.6$)$, while the adjusted $\mathrm{OR}$ was $0.8(95 \% \mathrm{Cl} 0.6$ to $0.9)$. Results regarding previous use of antidopaminergic drugs followed a similar risk pattern as for current use.

Conclusions: The lack of association between antidopaminergic drugs and acute pancreatitis after adjustment for confounding factors in this study suggests that the previously reported positive associations might be explained by confounding.

\section{ARTICLE SUMMARY}

\section{Article focus}

- Case reports and case series have suggested a link between use of antidopaminergic drugs and acute pancreatitis.

- The aim of this study was to clarify the relationship between use of antidopaminergic drugs and the risk of acute pancreatitis.

- We hypothesised that antidopaminergic drug use increases the risk of acute pancreatitis.

\section{Key messages}

- Our paper suggests that there is no association between antidopaminergic drug treatment and acute pancreatitis when adjusting for potentially confounding variables.

- Furthermore, our findings suggest that the previously reported positive association might be explained by confounding.

Strengths and limitations of this study

- Major strengths of the study are the populationbased design, utilising a nationwide register, counteracting recall and selection bias.

- Limitations include the relatively small number of acute pancreatitis cases during antidopaminergic exposure, lack of information on medication treatment during hospitalisation and lack of information about adherence to the prescribed medications.

\section{INTRODUCTION}

The incidence of acute pancreatitis is increasing for reasons that are not well understood. ${ }^{1}$ The main risk factors, that is, gallstones and excessive alcohol consumption, are present in approximately $40 \%$ and $30 \%$ of cases, respectively, while about $25 \%$ are of idiopathic (unknown) origin. ${ }^{1}$ There have been several case reports of acute pancreatitis during use of antidopaminergic medication. $^{2-18}$ Typically, the reported cases had initiated their medication within the 6 months before the onset of acute pancreatitis $^{5} \begin{array}{llll}7 & 9 & 12 & 15-19 \\ \text { and had recovered after }\end{array}$ withdrawing or switching antidopaminergic drug. ${ }^{5} 11 \quad 12$ 15-19 ${ }^{15}$ Some case reports have also reported recurrent pancreatitis after 
rechallenge of the suspected drug. ${ }^{8} 18$ Two possible biological mechanisms have been suggested. First, a hypersensitivity reaction might be occurring, an explanation that fits well with the observation that some cases have experienced recurrent pancreatitis at a much lower dose and much earlier at rechallenge and is in line with the well-described hypersensitivity reactions, mainly for clozapine with subsequent agranulocytosis or myocarditis. ${ }^{18}{ }^{20}$ Second, hypertriglyceridemia might be induced by the antidopaminergic drug, resulting in an accumulation of chylomicrons. ${ }^{17}$ However, the sparse epidemiological data available have not shown any clear positive association between antidopaminergic medication and the risk of acute pancreatitis. ${ }^{21} 22$ Larger epidemiological studies elucidating this possible association are warranted before any association can be established or excluded. The aim of this study was therefore to clarify the relationship between use of antidopaminergic drugs and the risk of acute pancreatitis in a large and population-based study, including adjustment for potential confounders. We hypothesised that antidopaminergic drug use increases the risk of acute pancreatitis.

\section{METHODS}

\section{Study design}

A Swedish nationwide population-based case-control study was performed in the period from 1 January 2006 to 31 December 2008. The source population was defined as all Swedish residents aged between 40 and 84 years. The Patient Register was used to identify cases with a first episode of acute pancreatitis. The Register of the Total Population was used to randomly select control subjects from the general population. Individual data on drug exposure among cases and control subjects were collected from the Prescribed Drug Register. Information on the highest achieved formal educational level was obtained from the Education Register. In order to censor for person-time no longer at risk of being diagnosed as having acute pancreatitis in the Patient Register, data regarding emigration and death were obtained from the Register of the Total Population and the Causes of Death Register, respectively. Additionally, censoring for any cancer was conducted through the Cancer Register. The unique personal identity number assigned to all Swedish residents was used to link individual information between the registers. ${ }^{23}$ The study has been approved by the Regional Ethical Review Board in Stockholm.

\section{Sources of data}

The Patient Register comprises information on all inhospital care and outpatient specialist care in Sweden, including codes for diagnoses (according to the 'International Classification of Diagnoses' (ICD)) and surgical procedures (according to the 'Nordic Classification of Surgical Procedures'). It has complete nationwide coverage of inpatient data since 1987 and complete outpatient specialist care data since 2001. ${ }^{24}$
The Prescribed Drug Register records all medications dispensed to individual patients since 1 July 2005, capturing the entire Swedish population of approximately 9 million inhabitants. ${ }^{25}$ This register contains data on drugs, including names of drug substances according to the Anatomical Therapeutic Chemical (ATC) classification. ${ }^{26}$ Additionally, it includes information about amount, dosage and date of expenditure and reimbursement, as well as patient data including age, sex and place of residence. The register lacks information regarding indication for treatment, over-the-counter drugs and drugs administered at hospitals during inpatient treatment.

The Cancer Register was set up in 1958, and since then every clinician, pathologist and cytologist in Sweden has been required to notify the National Board of Health and Welfare of every person diagnosed as having a new primary malignancy. The Cancer Register includes primary malignancies and certain benign tumours and precancerous lesions classified according to the ICD system. $^{27}$

The Causes of Death Register contains information on the dates and causes of all deaths of Swedish residents since 1952 , with $100 \%$ coverage of death dates. ${ }^{28}$

The Register of the Total Population contains individual characteristics of all Swedish residents since 1968 (100\% coverage), including sex, age, country of birth, marital status and place of residence. ${ }^{29}$

The Education Register was established by Statistics Sweden in 1985 and is annually updated with information on the highest formal education attained by each individual, from elementary to post-graduate level. ${ }^{30}$

\section{Case and control identification}

Cases were defined as individuals in the study cohort with a first time registered discharge diagnosis in the Patient Register of acute pancreatitis between 2006 and 2008. The code K85 in the 10th version of the ICD represented acute pancreatitis. Control subjects were randomly selected according to the principle of frequency-based density sampling, matched for age, sex and calendar year. Exposure was considered in relation to an index date assigned to each case and control participant. For case subjects, the index date was set to the date of admission for acute pancreatitis. For control subjects, the index date was a randomly assigned date within the study period. All potential case and control subjects with a previous cancer (apart from non-melanoma cancers of the skin) or any pancreatic disease (defined by the diagnosis codes K85, K86, K87 (ICD-10) and 577 (ICD-9)) recorded in the Patient Register before the study started were excluded.

\section{Exposure to antidopaminergic drugs}

Dispensed drug prescriptions of antidopaminergic drugs were identified by their specific ATC code (N05A) in the Prescribed Drug Register. Only exposure prior to the index date was considered. As drugs for longer term 
use typically are dispensed for 3-month periods in Sweden, we assumed that a prescription normally comprised 100 days of drug use and added a margin of 14 days. Thus, a single prescription of antidopaminergic drugs was assumed to last 114 days. Antidopaminergic drug use was defined as 'current', 'recent', 'past' or 'former', if the drug had been dispensed 1-114 days, 115-180 days, $181-365$ days or $1-3.5$ years before the index date, respectively. Previous use was categorised to enable us to study short-term effects and better evaluate potential confounding effects of concomitant diseases. Depot formulations were recalculated to fit the above categorisation. The absence of any prescription for antidopaminergic drugs was classified as 'non-use'. Two groups of antidopaminergic drugs were studied separately: (1) antiemetic/anxiolytics, including dixyrazine, levomepromazine, melperone and prochlorperazine, and (2) other antipsychotics, including fluphenazine, perphenazine, flupenthixole, thioridazine, chlorpromazine, haloperidol, pimozide, ziprasidone, aripiprazole, quetiapine, risperidone, paliperidone, clozapine and olanzapine. A separate analysis of the two most metabolically adverse antipsychotic drugs, clozapine and olanzapine, was also conducted. To facilitate comparison with previous studies, we also performed secondary analyses grouping the antidopaminergic drugs into atypical/typical antipsychotics and subdividing typical into high-, medium- and low-potency antipsychotics. Finally, to maximise power, we made an analysis including any antidopaminergic drug use in one group.

\section{Statistical analysis}

The RR of acute pancreatitis was estimated by unconditional logistic regression, which was used to calculate ORs with 95\% CIs. Adjustments were made in three models: (1) a crude model was based on the matching variables only, that is, sex, age (in 5-year age groups) and the three calendar years; (2) a multivariable model added (a) history of excessive alcohol consumption or disease related to alcohol, defined by the diagnosis codes E244, F10, G312, G621, G721, I426, K292, K70, O354 and T51 in ICD-10 or 291, 303, 305A, 357F, 425F, 535D, $571 \mathrm{~A}, 571 \mathrm{~B}, 571 \mathrm{C}, 571 \mathrm{D}$ and 980 in ICD-9, or use of antialcohol drugs defined by the ATC code N07BB and (3) a full multivariable model further added (b) chronic obstructive lung disease, defined by the diagnosis codes $\mathrm{J} 41, \mathrm{~J} 42, \mathrm{~J} 42$ and $\mathrm{J} 44$ in ICD-10 or 491, 492 and 496 in ICD-9, (c) ischaemic heart disease, defined by the diagnosis codes I20-I25 in ICD-10 or 410-413, 414A and $414 \mathrm{~W}$ in ICD-9, (d) obesity, defined by the diagnosis codes E66 in ICD-10 or 278A in ICD-9 or antiobesity drugs defined by the ATC code A08A, (e) diabetes, defined by the diagnosis codes E10-E14 in ICD-10 or 250 in ICD-9 or antidiabetic medication, defined by the ATC code A10, (f) opioid drug use, defined by the ATC code N02A, (g) gallstone disease, defined by the diagnosis codes $574,575 \mathrm{~A}$ or $575 \mathrm{~B}$ in ICD-9 or K80 and K81 in ICD-10, (h) educational level (divided into three categories of highest attained education: elementary school, secondary school, university and one category for missing data (2.3\% among cases and $1.6 \%$ among controls)), (i) marital status (categorised as married or not) and ( $\mathrm{j}$ ) a comorbidity score based on number of distinct medications. The comorbidity score was defined as the sum of unique 7-digit ATC codes dispensed during the 6 months prior to the index date and was categorised as $0-4,5-9,10-14$ or $\geq 15$ drugs. Presence of the listed diseases or conditions was determined by a recorded hospitalisation or outpatient visit according to the Patient Register since 1987 or a dispensed prescription between 1 July 2005 and the index date. In a subanalysis, the study period was restricted to 2007 and 2008 to account for the fact that the study participants, depending on their index date, had different follow-up times from the start of the Prescribed Drug Register on 1 July 2005. Hence, for some participants included throughout the entire study period, we could assess drug exposure only 1-180 days before the index date. In addition, the association between antidopaminergic medication and acute pancreatitis was studied among those with and without a previous hospital record of psychosis, defined by the diagnosis codes F20-F29 in ICD-10 or 295, 296, 297 and 298 in ICD-9. The SAS statistical software package EG 4.2 (SAS Institute) was used for the analyses.

\section{RESULTS}

\section{Study participants}

The study included 6161 cases of acute pancreatitis and 61637 control subjects. Characteristics of the study participants are presented in table 1 . The frequencybased sampling produced a similar age and sex (and calendar year) distribution among cases and control subjects. Among the cases, 78 had used antiemetic/ anxiolytic antidopaminergic drugs and 194 had used other antipsychotics. Current and previous use of antiemetic/anxiolytics, as well as other antipsychotics, was more common among cases than controls (table 1). All covariates presented in the Methods section were overrepresented among the case subjects compared with the control participants. This was especially evident for factors representing lifestyle. Diseases related to alcohol overconsumption were, for example, four times more common in cases than controls (table 1).

\section{Antiemetic/anxiolytics and risk of acute pancreatitis}

Table 2 presents the ORs for the association between antiemetic/anxiolytics use and other antipsychotic medication and the risk of developing acute pancreatitis. The ORs in the crude model, adjusting for the matching variables, indicated an increased risk among current users of antiemetic/anxiolytics $(\mathrm{OR}=1.9,96 \%$ CI 1.4 to 2.6) as well as for each of the categories representing previous use of antiemetic/anxiolytics (table 2). In the multivariable model, adjusting for alcohol-related comorbidity, the risk among current users of antiemetic/ 
Table 1 Characteristics of cases with acute pancreatitis and frequency-matched population control subjects in Sweden during the period 2006-2008

\begin{tabular}{|c|c|c|}
\hline & $\begin{array}{l}\text { Cases } \\
\text { Number (\%) }\end{array}$ & $\begin{array}{l}\text { Controls } \\
\text { Number (\%) }\end{array}$ \\
\hline Total & $6161(100.0)$ & $61637(100.0)$ \\
\hline \multicolumn{3}{|l|}{ Sex } \\
\hline Women & $2774(45.0)$ & $27745(45.0)$ \\
\hline Men & $3387(55.0)$ & $33892(55.0)$ \\
\hline \multicolumn{3}{|l|}{ Age group (years) } \\
\hline $40-44$ & $522(8.5)$ & $5226(8.5)$ \\
\hline $45-49$ & $533(8.7)$ & $5336(8.7)$ \\
\hline $50-54$ & $648(10.5)$ & $6480(10.5)$ \\
\hline $55-59$ & $749(12.2)$ & $7498(12.2)$ \\
\hline $60-64$ & $883(14.3)$ & 8830 (14.3) \\
\hline $65-69$ & $776(12.6)$ & $7760(12.6)$ \\
\hline $70-74$ & $702(11.4)$ & 7019 (11.4) \\
\hline $75-79$ & $689(11.2)$ & $6889(11.2)$ \\
\hline $80-84$ & 659 (10.7) & $6599(10.7)$ \\
\hline \multicolumn{3}{|c|}{ Use of other antidopaminergic drugs* } \\
\hline No use & $5967(96.9)$ & $60415(98.0)$ \\
\hline Current (0-114 days) & $108(1.8)$ & $807(1.3)$ \\
\hline Recent (115-180 days) & $14(0.2)$ & $79(0.1)$ \\
\hline Past (6-12 months) & $21(0.3)$ & $129(0.2)$ \\
\hline Former (>12 months) & $51(0.8)$ & $207(0.3)$ \\
\hline \multicolumn{3}{|c|}{ Use of antiemetic/anxiolytic antidopaminergic drugst } \\
\hline No use & $6083(98.7)$ & $61315(99.5)$ \\
\hline Current (0-114 days) & $36(0.6)$ & $201(0.3)$ \\
\hline Recent (115-180 days) & $10(0.2)$ & $22(0.0)$ \\
\hline Past (6-12 months) & $13(0.2)$ & $40(0.1)$ \\
\hline Former (>12 months) & $19(0.3)$ & $59(0.1)$ \\
\hline \multicolumn{3}{|c|}{ Alcohol-related diagnoses or drugs for alcoholism $\ddagger$} \\
\hline No & $5565(90.3)$ & $60030(97.4)$ \\
\hline Yes & $596(9.7)$ & $1607(2.6)$ \\
\hline \multicolumn{3}{|c|}{ Gallstone-related diagnoses $\S$} \\
\hline No & $5086(82.6)$ & $59102(95.9)$ \\
\hline Yes & $1075(17.4)$ & $2535(4.1)$ \\
\hline
\end{tabular}

Generic name for antidopaminergic drugs used time before index date.

*Fluphenazine, perphenazine, flupenthixole, thioridazine, chlorpromazine, haloperidol, pimozide, ziprasidone, aripiprazole, quetipaine, risperidone, paliperidone, clozapine and olanzapine. tDixyrazine, levomepromazine, melperone and proklorperazine. $\neq$ International Classification of disease ICD: E244, F10, G312, G621, G721, 1426, K292, K70, O354 or T51 in ICD-10 or 291, 303, $305 \mathrm{~A}, 357 \mathrm{~F}, 425 \mathrm{~F}, 535 \mathrm{D}, 571 \mathrm{~A}, 571 \mathrm{~B}, 571 \mathrm{C}, 571 \mathrm{D}$ or 980 in ICD-9 or drugs used for treatment of alcohol addiction.

SInternational Classification of disease ICD: K80 or K81 in ICD-10 or $574,575 \mathrm{~A}$ or $575 \mathrm{~B}$.

anxiolytics was attenuated ( $\mathrm{OR}=1.4,95 \%$ CI 1.0 to 1.9$)$, and in the full multivariable model, no increased risk for acute pancreatitis was observed for current use of antiemetic/anxiolytic ( $\mathrm{OR}=0.9,95 \%$ CI 0.6 to 1.2$)$.

\section{Other antipsychotic medication and risk of acute pancreatitis}

Correspondingly, current use of other antipsychotics was associated with an increased risk of acute pancreatitis in the crude model $(\mathrm{OR}=1.4,95 \%$ CI 1.1 to 1.6$)$. However, this risk increase disappeared after adjusting for confounding by alcohol-related comorbidity $(\mathrm{OR}=1.1$,
95\% CI 0.9 to 1.4 ). Each of the categories representing previous use of other antipsychotics showed a similar decrease in the ORs from the crude model to the fully adjusted model (table 2).

For current use of clozapine or olanzapine, the OR in the crude model was 1.6 (95\% CI 1.1 to 2.3 ) and decreased to 0.9 (95\% CI 0.6 to 1.4 ) in the full multivariable model.

Stratifying by sex did not reveal any major differences between the sexes (data not shown). When stratifying by age, the results for the group younger than 65 years were similar to those of the main analysis, but among those 65 years and older, the OR for current use was close to unity in both the crude and the fully adjusted model (data not shown). Among participants younger than 65 years, the group with a previous history of alcohol overconsumption had no increased risk of acute pancreatitis associated with current use of other antipsychotics, in either the crude or the full multivariable model (OR 0.8, 95\% CI 0.5 to 1.3 and OR $0.8,95 \%$ CI 0.5 to 1.2 , respectively). However, participants without such history of alcohol overconsumption rendered similar results as in the main analysis (data not shown). Individuals with a previous hospital record of psychosis had no increased risk for current use of other antipsychotics in the crude or full multivariable model (data not shown). However, participants with a previous hospital record of psychosis who had stopped other antipsychotic medication within the last 3 months were at an increased risk of acute pancreatitis after adjustment in the multivariable model (OR $4.095 \% 1.4$ to 11.2). Stratifying by calendar year (2006 and 2007-2008) rendered similar results as in the main analysis (data not shown).

Analysing cumulative dose of antidopaminergic drugs since start of the Drug Register in 1st July 2005 and risk of acute pancreatitis yielded the highest OR for the lowest quartiles of total amount of Daily Defined Dose (OR 2.0, 95\% CI 1.58 to 2.51) and the lowest OR for the $10 \%$ with the highest amount of Daily Defined Dose $(\mathrm{OR}=0.93,95 \%$ CI 0.72 to 1.21$)$ in the crude model. Adjustment for alcohol-related diseases attenuated the association for all categories of cumulative dose (data not shown).

Within 114 days before being admitted for acute pancreatitis, 1098 (18\%) of cases were hospitalised for other reasons at least once, within 60 days before and 30 days before the corresponding hospitalised cases were $850(14 \%)$ and $608(9.9 \%)$, respectively. The corresponding figures for hospitalisation in a psychiatric ward was $78(1.3 \%), 43(0.7 \%)$ and $26(0.4 \%)$, respectively. The median length of stay for somatic hospitalisations was 4.3 and 3 days for the three time intervals, respectively, and for psychiatric hospitalisations 4.3 and 2 days.

The secondary analyses of atypical antipsychotics and three potency groups of typical antipsychotics showed low or no risk increase except for former use of medium potency antipsychotics and a non-significant increased 


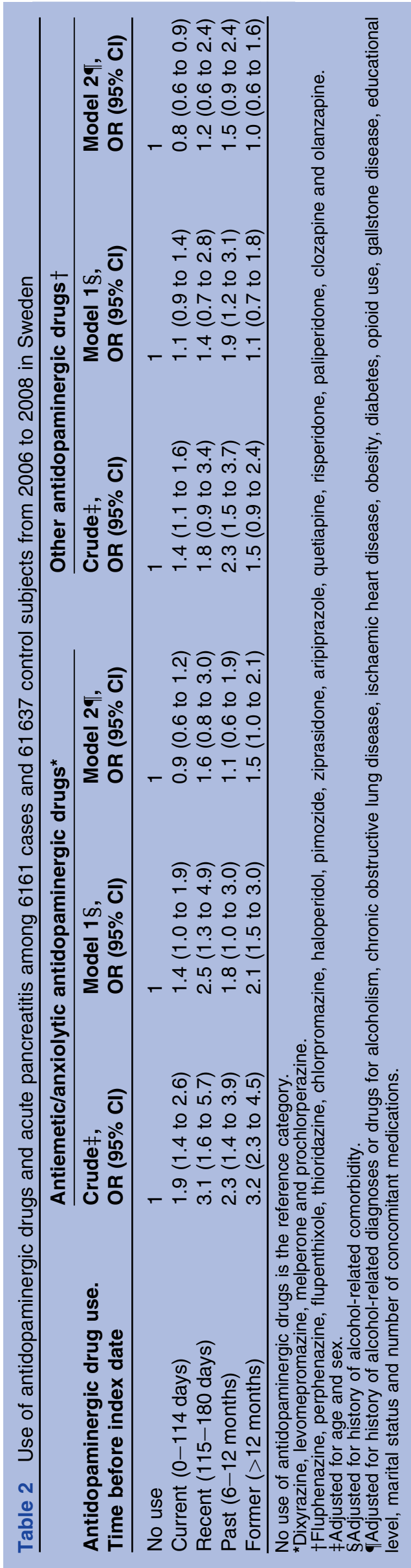

risk for recent use of low-potency antipsychotics (supplemental tables I and II). Use of any antidopaminergic drug was not associated with increased risk of acute pancreatitis.

\section{DISCUSSION}

This population-based study found no association between current use of antidopaminergic medication and acute pancreatitis after adjustment for potential confounding factors.

Among strengths of the study is the population-based design, utilising a nationwide register, counteracting recall and selection bias. Other advantages include the large sample size, the complete nationwide coverage of the exposure (antidopaminergic drugs) and the outcome (acute pancreatitis) and the adjustment for several potential confounding factors. Misclassification of the outcome in the Patient Register is a possible concern, but the high validity of the diagnosis of acute pancreatitis in this register has recently been shown by our group to have a positive predictive value of $98 \% .{ }^{31}$ There are, however, also several weaknesses. We regarded a dispensed prescription of antidopaminergic drugs as an exposure, but there was no information on whether the patients had actually taken their medication. This potential misclassification of the exposure would most likely be non-differential, that is, not associated with risk for future acute pancreatitis and thus, dilute the risk estimates towards null results. Additionally, there was no information on drugs administered during inhospital care and no information on indication for treatment. This might lead to a misclassification resulting in some of the patients with probably the highest doses of antidopaminergic drugs (being inpatient treated) being classified as not having been exposed to antidopaminergic drugs. However, as other hospitalisations prior to index hospitalisation for acute pancreatitis were not common and were of short duration, we consider this potential misclassification negligible. Moreover, detailed patient information is not available in registers, particularly regarding some potential confounding factors. It could, for instance, be possible that substantial alcohol consumption, smoking or obesity could not be captured with the variables retrieved from the national registers. Nevertheless, several potential confounding variables were adjusted for, and the results clearly show that confounding by these factors did occur and were seemingly sufficiently adjusted for. There is no reason to believe that misclassification of alcohol exposure would be a greater concern among the controls than the cases.

Use of antidopaminergic drugs in our study was associated with an increased risk of acute pancreatitis in the crude models, but after adjustment for known risk factors for acute pancreatitis, this association disappeared. The principal confounding effect seemed to be through alcohol, as the major decrease of the elevated risk of acute pancreatitis among users of 
antidopaminergic drugs was attenuated when adjusting for alcohol-related diagnoses and drug treatments for alcohol dependence. This confounding may be for two different reasons. First, antidopaminergic drugs are used as a non-addictive alternative to treat withdrawal, agitation and anxiety among patients with alcohol dependence. $^{32}$ Second, alcohol dependence is overrepresented among patients with severe mental illnesses, where antidopaminergic drugs often are the first-line treatment. ${ }^{33}$ Not all the increased risk disappeared after adjusting for alcohol; instead, the effect entirely disappeared only after further adjustment for additional known risk factors for acute pancreatitis, that is, chronic medical conditions, sociodemographic factors, obesity and gallstone-related diseases.

This study revealed no association between clozapine and olanzapine and the risk of acute pancreatitis. These two antipsychotic drugs are known to be particularly obesogenic, diabetogenic and with high liability of inducing lipid abnormalities. ${ }^{34-36}$ Yet, other studies have shown that obesity, as well as diabetes, might increase the risk of acute pancreatitis. ${ }^{37} 38$

Furthermore, in our study, patients with a previous hospital record of psychosis who had stopped using other antipsychotic medication within the previous 3 months had an increased risk of acute pancreatitis which remained after full adjustment. One possible explanation of this delay in the effect is that patients with a psychotic disorder are more non-adherent to pharmacotherapy when suffering from a comorbid alcohol abuse disorder. ${ }^{39}$ Thus, patients might have stopped their antipsychotic medication following a period of excessive alcohol intake, which subsequently may have led to an acute pancreatitis.

Before the analyses, we chose to divide antidopaminergic drugs into two groups according to the main clinical usage pattern. The antidopaminergic drugs in the group antiemetic/anxiolytics are typically used intermittently and in lower doses than other groups of antidopaminergic drugs. Notably, users of antiemetic/ anxiolytic had a higher crude risk of acute pancreatitis than users of other antipsychotics in our study, although they probably received lower total doses. However, one might speculate that if the antidopaminergic drugs in the antiemetic/anxiolytic group are used more often for treatment of withdrawal and anxiety in the post-detoxification phase from alcohol, than other antipsychotics, it would explain this discrepancy. Other studies have chosen to divide antidopaminergic drugs into conventional and atypical antipsychotics, ${ }^{13} 22$ but the rationale behind such categorisation has been criticised and recently has been proposed to be abandoned due to the great overall heterogeneity within these groups. ${ }^{40}$ However, for the sake of comparison with a recent Danish case-control study, ${ }^{22}$ we performed secondary analyses with the same categorisation of the antidopaminergic drugs. Former use of medium potency antipsychotics was associated with increased risk of pancreatitis, as was recent use of low-potency antipsychotics, even though non-significant. Nevertheless, none of the other time exposure categories were associated with a risk increase, and thus, it is possible that those two observations are chance findings. However, an actual effect cannot be entirely ruled out as similar results have been found previously and the CIs in the present study are relatively large. However, it is reassuring that, at least, no findings in the current use group approached significance and that there was an inverse relationship between cumulative DDD and risk of pancreatitis. Nevertheless, studies with larger numbers of patients exposed to these individual agents are needed to confirm our findings.

The case reports and case series indicating an association between antidopaminergic drugs and acute pancreatitis concerned the following compounds previously attributed as atypicals: clozapine, ${ }^{2} 101318$ olanzapine, ${ }^{3} 48^{8} 11-13 \quad 17$ quetiapine, ${ }^{7}{ }^{16}$ risperidone, ${ }^{5} 6 \quad 913$ aripiprazole and $^{15}$ ziprasidone ${ }^{19}$ and reported on the conventional antipsychotics haloperidol and chlorpromazine. ${ }^{13} 14$ However, relying simply on adverse reaction reports would probably exaggerate the possible risk of acute pancreatitis, as case reports have a limited capability to establish causality between drug use and disease outcome in the absence of re-exposure confirmation. There are few epidemiological studies available. A Swedish case-control study observed an overall increased risk for all phenothiazines. ${ }^{21}$ However, it included clomethiazole among the phenothiazines, a hypnotic compound which has been one of the firstline treatments for alcohol withdrawal, and adjustments for alcohol comorbidity were not made. A recent Danish pharmacoepidemiological study found an increased risk for acute pancreatitis among current users of low-potency conventional antipsychotics (mainly levomepromazine) even after having adjusted for alcoholrelated diagnoses, whereas former users of atypicals displayed a decreased risk. ${ }^{22}$ The contrasting findings to our study can probably be attributed to different methodology, where we might have reduced residual confounding by alcohol more completely, but it might still be present in the Danish study. This was accomplished in our study, as we used a much broader definition and inclusion of alcohol-related diagnoses, and we also included prescription of medications used to treat alcohol dependence.

In conclusion, this large population-based study with complete data on the exposures and outcome indicates that use of antidopaminergic drugs is not associated with any increased risk of acute pancreatitis after proper adjustment for confounding by known risk factors for pancreatitis. Therefore, any acute pancreatitis among antidopaminergic-treated patients might not be attributed to the antidopaminergic pharmacotherapy per se but instead to the classical risk factors of acute pancreatitis such as excessive alcohol intake, gallstones and obesity. 
Author affiliations

${ }^{1}$ Department of Neuroscience, Unit of Psychiatry, Uppsala University, Uppsala, Sweden

${ }^{2}$ Centre for Pharmacoepidemiology (CPE), Department of Medicine, Solna, Karolinska Institutet, Stockholm, Sweden

${ }^{3}$ Upper Gastrointestinal Research, Department of Molecular Medicine and Surgery, Karolinska Institutet, Stockholm, Sweden

${ }^{4}$ Division of Cancer Studies, King's College London, London, UK

${ }^{5}$ Section of Upper Gastrointestinal Surgery, Gastrocenter, Karolinska

University Hospital, Stockholm, Sweden

Contributors RL came up with the idea for the study. All authors participated in designing the study protocol. FM constructed and maintained the database. $\mathrm{RL}$ conducted the statistical analyses. RB and RL drafted the manuscript. All authors revised the manuscript. RL is the guarantor for the study.

Funding The study was partly supported by unrestricted grants from Swedish Research Council (SIMSAM) and by unrestricted grants from Uppsala County Council (ALF)

Disclosures All authors have completed the Unified Competing Interest form at http://www.icmje.org/coi_disclosure.pdf (available on request from the corresponding author) and declare that (1) none of the authors have support from any company for the submitted work; (2) none of the authors have any relationships with companies that might have an interest in the submitted work in the previous 3 years; (3) their spouses, partners or children have no financial relationships that may be relevant to the submitted work and (4) none of the authors have non-financial interests that may be relevant to the submitted work

Competing interests None.

Ethics approval The study has been approved by the Regional Ethical Review Board in Stockholm.

Provenance and peer review Not commissioned; externally peer reviewed.

Data sharing statement No additional data available.

\section{REFERENCES}

1. Appelros $S$, Borgstrom $A$. Incidence, aetiology and mortality rate of acute pancreatitis over 10 years in a defined urban population in Sweden. Br J Surg 1999;86:465-70.

2. Bayard JM, Descamps OS, Evrard S, et al. Case report: acute pancreatitis induced by Clozapine. Acta Gastroenterol Belg 2005;68:92-4.

3. Belli H, Sertbas $Y$, Bayik Y. Olanzapine-induced diabetes due to pancreatitis. Indian J Gastroenterol 2005;24:273.

4. Bracamonte JD, Underhill M, Sarmiento P. Acute pancreatitis associated with lisinopril and olanzapine. Am J Health Syst Pharm 2010;67:214-16.

5. Cordeiro Q Jr, Elkis H. Pancreatitis and cholestatic hepatitis induced by risperidone. J Clin Psychopharmacol 2001;21:529-30.

6. Ghio L, Fornaro G, Rossi P. Risperidone-induced hyperamylasemia, hyperlipasemia, and neuroleptic malignant syndrome: a case report. $J$ Clin Psychopharmacol 2009;29:391-2.

7. Gropper D, Jackson CW. Pancreatitis associated with quetiapine use. J Clin Psychopharmacol 2004;24:343-5.

8. Hagger R, Brown C, Hurley P. Olanzapine and pancreatitis. $\mathrm{Br} J$ Psychiatry 2000;177:567.

9. Hanft A, Bourgeois J. Risperidone and pancreatitis. J Am Acad Child Adolesc Psychiatry 2004;43:1458-9.

10. Huang YJ, Lane HY, Liao $\mathrm{CH}$, et al. Recurrent pancreatitis without eosinophilia on clozapine rechallenge. Prog Neuropsychopharmacol Biol Psychiatry 2009;33:1561-2.

11. Kahn D, Bourgeois JA. Acute pancreatitis and diabetic ketoacidosis in a schizophrenic patient taking olanzapine. J Clin Psychopharmacol 2007;27:397-400.

12. Kerr TA, Jonnalagadda S, Prakash C, et al. Pancreatitis following olanzapine Therapy: a report of three cases. Case Rep Gastroenterol 2007;1:15-20.

13. Koller EA, Cross JT, Doraiswamy PM, et al. Pancreatitis associated with atypical antipsychotics: from the Food and Drug Administration's MedWatch surveillance system and published reports. Pharmacotherapy 2003;23:1123-30.

14. Laghate VD, Gupta SB. Acute pancreatitis and diabetic ketoacidosis in non-diabetic person while on treatment with sodium valproate, chlorpromazine and haloperidol. J Assoc Physicians India 2004;52:257-8

15. Lattanzi L, Casamassima F, Brunetto M, et al. Asymptomatic hyperamylasemia and hyperlipasemia associated with aripiprazole. J Clin Psychopharmacol 2009;29:504-6.

16. Rashid J, Starer PJ, Javaid S. Pancreatitis and diabetic ketoacidosis with quetiapine use. Psychiatry (Edgmont) 2009;6:34-7.

17. Rossor AM, Leech N, Neely RD. Olanzapine-induced chylomicronemia presenting as acute pancreatitis. J Clin Psychopharmacol 2007;27:395-6.

18. Sani G, Kotzalidis GD, Simonetti A, et al. Development of asymptomatic pancreatitis with paradoxically high serum clozapine levels in a patient with schizophrenia and the CYP1A2*1F/1F genotype. J Clin Psychopharmacol 2010;30:737-9.

19. Yang SH, McNeely MJ. Rhabdomyolysis, pancreatitis, and hyperglycemia with ziprasidone. Am J Psychiatry 2002;159:1435.

20. Kilian JG, Kerr K, Lawrence C, et al. Myocarditis and cardiomyopathy associated with clozapine. Lancet 1999;354:1841-5.

21. Blomgren KB, Sundstrom A, Steineck G, et al. A Swedish casecontrol network for studies of drug-induced morbidity-acute pancreatitis. Eur J Clin Pharmacol 2002;58:275-83.

22. Gasse C, Jacobsen J, Pedersen L, et al. Risk of hospitalization for acute pancreatitis associated with conventional and atypical antipsychotics: a population-based case-control study. Pharmacotherapy 2008;28:27-34.

23. Ludvigsson JF, Otterblad-Olausson $\mathrm{P}$, Pettersson BU, et al. The Swedish personal identity number: possibilities and pitfalls in healthcare and medical research. Eur J Epidemiol 2009;24:659-67.

24. Rosen M. National Health Data Registers: a Nordic heritage to public health. Scand J Public Health 2002;30:81-5.

25. Wettermark B, Hammar N, Fored C, et al. The new Swedish Prescribed Drug Register-Opportunities for pharmacoepidemiological research and experience from the first six months. Pharmacoepidemiol Drug Saf 2007;16:726-35.

26. WHO Collaboration Centre for drugs Statistics methodology. http:// www.whocc.no/atcddd/ (accessed 13 Oct 2011).

27. Barlow L, Westergren $\mathrm{K}$, Holmberg $\mathrm{L}$, et al. The completeness of the Swedish Cancer Register: a sample survey for year 1998. Acta Onco 2009;48:27-33.

28. Socialstyrelsen (The National Board of Health and Welfare). Causes of Death Statistics-Quality Aspects [Dödsorsaksstatistik-Historik, produktionsmetoder och tillförlitlighet]. Sweden, 2010. http://www. socialstyrelsen.se/publikationer2010/2010-4-33 (accessed 13 Oct 2011).

29. Statistics Sweden. Main Outlines for Organization of Population Statistics. 2007. http://www.scb.se/statistik/_publikationer/ BE0101_2007A01 BR 19 BE0108TAB.pdf (accessed 13 Oct 2011)

30. Statistics Sweden. The Swedish Register of Education. 2004. www. scb.se/statistik/UF/UF0506/Produktbeskrivning_short_English_ UF0506 20040101r.doc. (accessed 13 Oct 2011).

31. Razavi D, Ljung R, Lu Y, et al. Reliability of acute pancreatitis diagnosis coding in a national patient register: a Validation study in Sweden. Pancreatology 2011;11:525-32.

32. Mayo-Smith MF. Pharmacological Management of alcohol withdrawal. JAMA 1997;278:144-51.

33. Regier DA, Farmer ME, Rae DS, et al. Comorbidity of mental disorders with alcohol and other drug abuse. JAMA 1990;264:2511-18.

34. Allison DB, Mentore JL, Heo M, et al. Antipsychotic-induced weight gain: a comprehensive research synthesis. Am J Psychiatry 1999;156:1686-96

35. Wu RR, Zhao JP, Zhai JG, et al. Sex difference in effects of typical and atypical antipsychotics on glucose-insulin homeostasis and lipid metabolism in first-episode schizophrenia. J Clin Psychopharmacol 2007;27:374-9.

36. Leucht $\mathrm{S}$, Komossa $\mathrm{K}$, Rummel-Kluge $\mathrm{C}$, et al. A meta-analysis of head-to-head comparisons of second-generation antipsychotics in the treatment of schizophrenia. Am J Psychiatry 2009; 166:152-63.

37. Blomgren KB, Sundström A, Steineck G, et al. Obesity and treatmen of diabetes with Glyburide may both Be risk factors for acute pancreatitis. Diabetes Care 2002;25:298-302.

38. Girman CJ, Kou TD, Cai B, et al. Patients with type 2 diabetes mellitus have higher risk for acute pancreatitis compared with those without diabetes. Diabetes Obes Metab 2010;12:766-71.

39. Novick D, Haro JM, Suarez D, et al. Predictors and clinica consequences of non-adherence with antipsychotic medication in the outpatient treatment of schizophrenia. Psychiatry Res 2010;176:109-13.

40. Leucht S, Corves C, Arbter D, et al. Second-generation versus firstgeneration antipsychotic drugs for schizophrenia: a meta-analysis. Lancet 2009;373:31-41. 